\title{
Energy generation by waste incineration: the management of impregnated wood
}

\author{
R. Sjöblom \& J. Kumpiene \\ Waste Science and Technology, Luleå University of Technology, Sweden
}

\begin{abstract}
Landfilling of organic waste is no longer allowed in Sweden. Instead, essentially all such waste is being recycled, and about half of it goes to incineration which accounts for about $10 \%$ of the total need for heating of buildings. Incineration implies destruction of potentially harmful constituents in the waste, but does not destroy contaminant elements such as arsenic which almost exclusively originates from impregnated wood. Methods for identification of chromium, copper and arsenic in such wood are analysed as well as techniques for sorting it into two categories. If incinerated separately, these can give rise to ash with $\mathrm{Cr}$, $\mathrm{Cu}$ and $\mathrm{As}$, and ash with only $\mathrm{Cu}$. The former ash has a small volume and can be stabilized/landfilled at a qualified facility, and the latter ash might be used for beneficiation of $\mathrm{Cu}$. In addition, the contamination by As, especially, in other fuels will be small and consequently also in the ash, thus facilitating its use. It is found that such sorting may be achieved using visual inspection as well as X-ray fluorescence (XRF), whilst use of reagents does not appear to offer any advantage over these two. Both methods are already in industrial use in Sweden, thus proving the feasibility of segregation and stabilization of contaminants in impregnated wood.
\end{abstract}

Keywords: impregnated wood, sorting, arsenic, XRF, incineration, ash.

\section{Background}

\subsection{The role of incineration in the Swedish energy system}

The utilization of waste as a source for generation of heat and electricity is intimately related to the policy and practice for waste disposal. Some ten years ago, legislation in Sweden was gradually implemented so that organic and 
degradable waste, including domestic waste, could no longer be subjected to landfilling. The main reason for this was not to jeopardize future stability.

Today, the domestic waste is sorted into two main fractions, one to be incinerated comprising about $50 \%$ of the total, and another to be composted. The development may be illustrated by the following figures: around 1.4 million tonnes of domestic waste was landfilled in the year 1994, while the corresponding figure for the year 2013 is only 0.033 million tonnes. Thus 2.34 million tonnes of domestic waste was incinerated in Sweden in the year 2013 [1]. The population of Sweden is close to ten million inhabitants.

Moreover, the incinerators that burn domestic waste also burn industrial waste, and thus the total volume incinerated amounts to 5.3 million tonnes. In addition, industrial residues are also combusted/incinerated at other industrial facilities (e.g. paper mills), sometimes feeding not only their own facilities but also the district heating grids. Such additional incineration is not included in most of the statistics provided here.

Incineration is congruent with the ban on landfilling. During combustion, degradable waste is converted to inorganic forms which do not decay to generate methane or differential settlements. The weight of the waste is decreased to around $18-25 \%$, and the decrease in volume is much higher.

Internationally, the public is often concerned about emissions, but this is not much of an issue in Sweden. The main reasons for this is that the legislation is very strict and that the utilities comply conscientiously.

In terms of economics, the value of the destruction service typically equals that of the generation of the energy, i.e. the added value at an incineration plant may be twice as high as that of a plant burning pure biofuels. Incinerators have high investment costs compared to other combustion facilities, and thus typically provide the base load whilst combustion of biofuels has a lower investment threshold, and can thus be used primarily when the weather is cold.

Generally, Sweden is a rather cold country with needs for heating that extend throughout around nine months of the year, depending on location. In comparison with other countries, this calls for high investment costs, efficient facilities and inexpensive fuels (or preferably fuels that one is paid to receive).

As a consequence, Sweden imports around 0.83 million tonnes of domestic waste and this share is increasing.

In the year 2013, the incinerators that burn domestic and other waste generated a total of $15.6 \mathrm{TWh}, 13.8$ of which was heat and 1.8 was electricity [1].

The heat was fed into district heating grids and, according to [2], this comprised $19 \%$ of a total of district heating of $55 \mathrm{TWh}$. District heating in Sweden covers around half of the total need for domestic as well as professional purposes. The consumption of district heating is roughly as follows [2]:

$\begin{array}{llll}\text { Offices and similar } & 30 \% & \text { Houses for } 1-2 \text { families } & 10 \% \\ \text { Industry } & 10 \% & \text { Apartment buildings } & 50 \%\end{array}$

Further details on the Swedish energy system can be found in [3]. 


\subsection{The metabolism of an incineration system}

It is important to keep in mind that incineration comprises not only thermochemical energy generation but also the handling and conversion of matter. This may be referred to as the metabolism of incineration $[3,4]$.

About 1.7 million tonnes of ash are generated in Sweden each year, most of which originates from incineration. It is apparent from the above that the energy generated from pure biofuels is considerably larger than that from waste, but since the ash content is low in pure biofuels, it is the ashes from incineration (of waste) that dominate substantially in terms of volume.

A lot of waste contains contaminant elements which are concentrated in the ash during the combustion process. It is important, from a health and environment point of view, how these elements are managed. Preferably, the waste is sorted initially with regard to such elements, and the different ashes obtained are dealt with separately. With such an approach, only a minor fraction of the ash would need stabilization and space in a landfill for hazardous waste. Conversely, low levels of contaminants, frequently together with selfstabilization, constitute important prerequisites for ash beneficiation or comparatively inexpensive landfilling.

Since most of the potentially hazardous compounds are destroyed during the incineration process, segregation in the present context refers mainly to contaminant elements, i.e. elements that may form compounds that could potentially be detrimental to health and the environment.

Similarly, stabilization in the present context should be understood as the process by which contaminant elements may be converted into such forms that render them inaccessible with regard to any reasonably realistic potential detrimental impact on health and the environment.

Some examples of process steps that may provide opportunity for segregation and stabilization are as follows:

- $\quad$ Sorting of the waste at the source;

- Sorting of the waste at the recycle plant;

- Separation of elements during incineration;

- Separation of elements in the ask;

- Stabilization of ash, including ageing in contact with moisture and air (oxygen and carbon dioxide).

Of course, efficient approaches with regard to segregation and stabilization vary for different elements. A substantial experience in this regard has been accumulated in conjunction with classification of a large number of residues from incineration from a majority of the facilities in Sweden $[5,6]$. It has been found that the potential hazard of an ash is in most cases dominated by the presence of the elements arsenic and lead.

These elements are quite different with regard to their chemical properties, their forms of occurrence and their distribution in the waste fuel. Arsenic originates almost exclusively from old impregnated wood, and such wood constitutes only a small fraction of all the waste fuel. As will be illustrated further below, arsenic is also treacherous with regard to its forms of occurrence. 
Lead is much more widely distributed in the waste. It is presumably more predictable in its behaviour in ash with regard to stabilization. It thus appears that, pertinent strategies for arsenic should include sorting, preferably at the source, whilst different approaches may be feasible for lead.

Arsenic as well as copper and chromium, and to some extent also zinc, have been studied in a recently completed project on impregnated wood carried out on commission by Avfall Sverige - Swedish Waste Management [7]. Lead and its stabilization by ageing ash in contact with moist air is presently being studied on commission by Avfall Sverige - Swedish Waste Management and Jönköping Energi AB.

The present paper deals with wood impregnated with arsenic, and to some extent also chromium and copper, and it is intended that the work on lead will be presented at the upcoming Waste Management 2016, 8th International Conference on Waste Management and the Environment. Thus, the present work relates to segregation whilst the planned paper refers to stabilization.

\section{Purpose and scope}

The purpose and scope of the present work is to compile a knowledge base that can be:

- used by the utilities in their decisions on a pertinent strategy with regard to impregnated wood waste, and

- used as a support in the implementation of such decisions.

In order for the knowledge base to serve such purposes, it needs to include the following:

- The health and environment properties of the contaminant elements in incinerator fuel containing impregnated wood as well as the corresponding ash.

- The properties of impregnated wood in question.

- The consequences of landfilling impregnated wood waste (as an alternative to incineration).

- The possibilities of measuring the levels of the contaminants in the wood, qualitatively and quantitatively.

- The possibilities to sort the impregnated wood with regard to the various contaminant elements of interest.

- The possible fate for the various fractions of ash generated from the different fractions of sorted impregnated wood waste fuel.

\section{Chromium, copper and arsenic in fuel and ash}

\subsection{Some properties of chromium, copper and arsenic}

\subsubsection{Chromium}

Chromium in soil occurs almost exclusively as chromium-III. Chromium can also have oxidation number VI, and at high $\mathrm{pH}$-values together with contact with oxygen in the air, chromium-III may oxidize to chromium-VI. 
Chromium-VI is not thermodynamically stable at high temperatures, and consequently it is expected that chromium in fresh ash has mainly the oxidation number III. It might be suspected that when ash is contacted with water, and in view of the high $\mathrm{pH}$ obtained in this process, that chromium in ash would oxidize to chromium-VI. Such a reaction would be expected to increase the leaching of chromium substantially since chemical substances containing chromium-VI are often very soluble in water. In concordance, there is usually some chromium in the leach water, but rarely more than a few percent of the total chromium. Thus, chromium in ash oxidizes only marginally in most cases.

The reason for this is that ash usually contains a large surplus of iron in comparison with the content of chromium. Chromium-III and iron (iron-II as well as iron-III) have closely the same ionic radii. Therefore, chromium in ash is stabilized as chromium-III even though the $\mathrm{pH}$ may be high $[6,8]$.

Contrary to what may be the case for chromium-VI, chromium-III in ash does not give rise to any hazardous properties. Chromium-III is e.g. the component in the stainless steel used in our kitchens that makes it resistant to corrosion.

\subsubsection{Copper}

Copper in ash usually has the valence II, and in this form it is not expected to give rise to any hazardous properties of the ash. For example, most of our domestic water pipes are covered on the inside with oxide-hydroxide-carbonatesulphate-chloride of copper-II. In addition, copper-II has closely the same ionic radius as iron-II/III, and is stabilized in solid solution with iron, similarly to chromium.

Moreover, copper possesses some rather unique chemical properties in that it (through its ligand field properties) has a tendency to form planar quadratic complexes. As a consequence, copper in nature often forms organic complexes. This makes it more mobile than many other elements.

\subsubsection{Arsenic}

Arsenic in soil usually has the oxidation numbers III or V, and each of these have different preferences with regard to chemical bonding.

Arsenic- $\mathrm{V}$ forms ionic complexes similar to phosphorus- $\mathrm{V}$. The availability to the water phase may be related to the speciation of phosphorus and calcium, since arsenate ions may go into solid solution with phosphate ions which, in turn, may be stabilized by the surplus of calcium that usually exists in ash (to form apatite, or similar).

Arsenic-III has closely the same ionic radius as iron-II/III, and is probably stabilized by the surplus of iron that is usually present in ash. Arsenic contaminated soil is sometimes treated with elementary iron in order for a surplus of reactive poorly crystalline iron oxide to be generated or ensured [9]. However, if the conditions are too reducing and/or acidic, then the availability of arsenic to the pore/leach water may increase [10]. Details on this is beyond the scope of the present work, and the reader is referred to Reference [11] for further information on the use of soil amendments. The book also contains information on soil chemistry in general. 
However, two further aspects of arsenic behaviour in soil and ash ought to be mentioned since they might be of importance in conjunction with landfilling:

- Inorganic arsenic can become metabolized biologically to volatile compounds such as methylated species, including trimethylarsine with the chemical formula $\mathrm{As}\left(\mathrm{CH}_{3}\right)_{3}$.

- Inorganic arsenic can react abiotically with particles rich in elemental aluminium to form arsine $\mathrm{AsH}_{3}$.

According to [12], section 4.3.1, "almost every organism" will methylate arsenic in oxide form. However, section 8.3.2, states that "no estimates of these arsanes have been made, but the totals are likely to be substantial". Generally, the biogenic generation of volatile arsenic compounds appears to be largely forgotten today, except in conjunction with phytoremediation, and hardly any references have been found in the literature on waste disposal.

However, a little over a century ago, arsenic was widely used in various consumer products. As the technique for chemical analysis of arsenic became developed (the arsenic mirror) (see e.g. Figure 16-4 in [13]), it was found that arsenic did spread from the products and into the bodies of humans. For a number of years, it was taken for granted that the transfer took place through dust, but Hamberg [14] found that arsenic actually occurred in the form of a gas, since air-borne arsenic could be absorbed in a gas wash bottle but not on a filter. Eventually, this discovery lead to a ban on arsenic in consumer products.

A few decades later, biogenic volatilization of heavy elements had obviously become forgotten, since mercury was introduced on a large scale for protection of planting grain against microorganisms. In this latter case, methyl mercury became volatilized. It accumulated in the food chain so that certain fish, for example, could no longer be eaten by humans.

There is also an inorganic pathway for volatile arsenic. Ash from incineration can contain small particles of elemental aluminium. Under the alkaline conditions formed when the ash is moistened, aluminium reacts with water to form hydrogen gas. The generation is usually surprisingly slow and extends over many days. Scanning electron microscopy data in [15] can be reinterpreted together with thermodynamic literature data on oxidation of aluminium and silicon to the conclusion that molten aluminium might have reduced silicon in oxide form to form an aluminium-silicon alloy with a protecting layer rich in silicon dioxide.

As will be dealt with in Section 4.2.2, arsenic in a sample can be quantitatively converted to arsine by the addition of zinc powder. Reference [16] shows that such a conversion can be achieved quantitatively also by using elemental aluminium.

It should be assumed that arsenic is poisonous and hazardous in all its forms of occurrence. However, different forms may have quite different properties. It may be noted that (at least many) substances containing arsenic not only have acute effects but are also carcinogenic. 


\subsection{Impregnated wood}

A number of chemical products have been used historically in order to protect wood from degradation by microorganisms. For simplicity, the present paper focuses on the main agents used during the past somewhat more than fifty years which are based on chromium, copper and arsenic (CCA, so-called Chromated Copper Arsenate), but details can be found in [7].

In Sweden, CCA impregnated wood has, in principle, been used only in outdoor applications. The use peaked around 1990 after which our Swedish Chemicals Agency stated that impregnated wood should be used only in applications where the wood is in permanent contact with soil or water. Further measures were taken in the year 2008 when the Agency ruled that CCA impregnated wood could only be used in a limited number of professional applications. Otherwise, wood was to be impregnated using agents that contain only copper. Moreover, the depth of impregnation was differentiated with respect to the intended use. Some wood was impregnated throughout (except for heartwood which does not accept impregnation), whilst another class of wood was impregnated only to a depth of a number of millimetres.

It is difficult to provide any detailed figures on inflow of impregnated wood to Sweden, and one major difficulty in this regard is that the export in many cases has been larger than the consumption. Nonetheless, around 400000 cubic metres per year is an appropriate order of magnitude, and it should be considered that the agent used as well as the procedure has changed over time as already described above.

The figures for the volumes of impregnated wood in the waste are even more uncertain. It has been estimated [7] that the impregnated wood separated at the source amounts to around 22000 tonnes per year, and that the amount that has not been thus separated may be of around the same magnitude.

It appears that the developments have been similar in other countries, including the United States.

The CCA impregnation agent was designed to form insoluble compounds in the wood, but it has nonetheless been estimated that such impregnated wood loses around $10 \%$ of its chromium and $30 \%$ of its copper and arsenic during the course of its life in service. It can be assessed, based on these estimates, that spent CCA impregnated wood contains around $1190 \mathrm{ppm}$ of chromium, 1060 ppm of copper and $1400 \mathrm{ppm}$ of arsenic.

CCA impregnated wood may have a profound influence on the composition of the ash. Ash from pure CCA impregnated wood will have rather high levels of chromium, copper and arsenic since such wood, which is void of bark and branches, has a very low ash content, perhaps only around $0.5 \%$. Arsenic in incinerator ash originates almost entirely from CCA impregnated wood. On average, copper contributes by only around $1 \%$ of the total copper in incinerator ash, and chromium by around $10 \%$. 


\subsection{Landfilling versus incineration}

It has already been mentioned above that since a number of years, Sweden has decided not to landfill organic matter in order not to jeopardize long-term stability of a landfill. Europe is presently also heading in this direction, but worldwide, organic matter, including wood, is landfilled for the most part.

It might be pertinent at this stage to ask oneself if exemption might be warranted for CCA impregnated wood, in view of the content of especially arsenic, and the concomitant increase of contaminant elements in the ash. However, contaminants in impregnated wood need attention also in conjunction with landfilling, since they may leach out from the wood over time, see [17] as well as Section 3.2 above.

Another aspect regarding landfilling is the generation of volatile arsenic compounds that might reach the biosphere together with the landfill gas (methane and carbon dioxide). No reference has been found on this issue, and it therefore appears to be difficult to rule out. Such generation might develop over time since microorganisms can develop resistance against arsenic.

Helsen and Van den Bulck [18] maintains that "A disadvantage of incineration is that it generates heat that has to be used immediately or converted to electricity (efficiency is relatively low) instead of producing a secondary fuel", and therefore proposes low temperature pyrolysis or high temperature gasification in spite of the fact that the authors realize that such techniques "still have to be proven". Fortunately for us in Sweden, such heat is just what we need and our incinerators are very capable of burning impregnated wood (see Section 1).

\section{Techniques for analysis and sorting}

\subsection{Introduction}

Sorting of waste before incineration is a relatively complex industrial activity from a systems analysis point of view, since various variables are interdependent. For instance, one important experience is that it is rarely a good idea to just accumulate waste and dump it at the recycling plant in order for it to be sorted there. In such cases, the subsequent sorting can become very timeconsuming and correspondingly expensive for the customer. Instead, waste is preferably as well as typically sorted at the site where it is generated, and then added to the different input categories at the recycling plant.

The task of separating impregnated wood from other waste fuel is therefore not such a formidable task as might otherwise be perceived. Moreover, wood impregnated with an agent containing copper has a clear green colour that is easily recognized unless the wood has been painted or severely weathered. However, if a high quality in the sorting is to be achieved, then the odd pieces of impregnated wood in the ordinary wood categories need to be identified and removed. Odd pieces of un-impregnated wood in the fraction for impregnated is not of similar significance. 
The sorting becomes more complicated if CCA-impregnated wood is to be separated from that containing only copper.

Important clues and indications can be obtained by various means, including shape of the pieces and likely previous use, visual inspection with or without uncovering the surface layer or internal part of the wood, use of reagents for chromium, copper and arsenic, and use of X-ray fluorescence techniques.

The experience so far at the Swedish facilities is clearly that it is a combination of methods that should be used, and thus, the question is what combinations are appropriate in which situations.

\subsection{Methods for identification and measurements}

\subsubsection{Visual inspection}

Visual inspection includes identification of the probable type of structure in which a piece of wood has served. Typically, one piece out of many of such a structure is examined more closely with regard to colour on aged and fresh surfaces, sometimes including a check if all of the splint in the wood has been impregnated (if not so, then it is likely that the impregnated agent used contained only copper). Experienced operators can often differentiate between the CCA and copper impregnated wood since the colour is somewhat different, but such differentiation might not be very reliable due to variations in the wood.

\subsubsection{Reagents}

Various reagents are commercially available for qualitative analysis of chromium, copper and arsenic in wood. Those for chromium and copper can be applied e.g. by a brush or a spray bottle onto the surface of the wood, and turn coloured within a few seconds to a minute when the element in question is present.

The presence of arsenic can be investigated using a test kit for determination of arsenic in water according to the March method [19]. In this test, the water sample is made acidic, after which zinc powder is added. The arsenic is then converted to arsine, which is volatile and can be brought to react with mercury bromide to form $\mathrm{AsH}_{2} \mathrm{HgBr}$ which is coloured. In order for the arsenic to become waterborne, the sample will have to be turned into sawdust. The analysis is said to take around 45 minutes [19].

It is very doubtful if this method should be used anywhere but in a laboratory and by qualified chemists since arsine is being generated.

\subsubsection{X-ray fluorescence}

$\mathrm{X}$-ray fluorescence (XRF) is based on the principle that an X-ray beam dislocates inner electrons after which other electrons replace them and emit photons (X-rays) having energies characteristic of the element in question.

Measurements can be carried out using a hand-held instrument, and takes from one to a few tens of seconds depending on the purpose. The area measured is small and several measurements may be required for any one piece. 
Qualitative measurements in the field can be carried out with only modest arrangements. Quantitative measurements require control of e.g. moisture, density and geometry as well as calibration.

Several elements can be determined in one measurement. The cost of an instrument is on the order of USD 30,000. Operators need to be properly introduced to how to use the instrument, not least in view of the potential hazard associated with the X-ray beam.

\subsubsection{Experience}

The choice of method is highly dependent on local prerequisites at each facility, such as e.g., whether or not more elaborate sorting might actually reduce costs, or give rise to improvements of the fuel mix (caloric value, ash and moisture contents, etc).

The approach at Halmstad Energi och Miljö AB is to use visual inspection together with a highly skilled and trained staff. The experience is that this approach will reach far in terms of separation, and considerably further than anticipated by one of the authors before visiting the facility.

$\mathrm{XRF}$ is being used for the fuel incinerated at Söderenergi AB. Their suppliers use it for qualitative measurements during the sorting, and the plant itself uses it for quantitative measurements when fuel is being delivered. The method has been applied to a large number of elements. In this way, Söderenergi has been able to qualify not only its bottom ash but also its fly ash as non-hazardous waste, and consequently achieved the advantages of supplying their ashes for use in geotechnical constructions or for depositing them at a landfill for nonhazardous waste.

The approach taken by Söderenergi is equally applicable to impregnated wood.

Reagents have hardly been used in Sweden, or perhaps not at all. It appears that they offer little advantage over the methods applied.

\section{Discussion and conclusions}

This paper focuses on sorting of impregnated wood after the waste has been generated but before it is incinerated. The main reason for not including surveying before demolition or washing of ash is that such techniques are used to a relatively small extent and that the issues related to impregnated wood might only add marginally to any motivation for such studies. As already mentioned, landfilling of impregnated wood is not an option in Sweden since we have a policy against landfilling easily degradable material. Another reason is that there may be some uncertainty regarding the long-term fate of arsenic, especially with regard to volatile species.

Separation of CCA-impregnated wood together with burning it separately would lead to small volumes of contaminated ash that would have to be stabilized and/or landfilled at a qualified facility. This is very preferable in comparison with dilution with other ash such as is the case with unsorted wood waste. Also, there exists some uncertainty regarding the possibility of generation 
of arsine in the case of mixing arsenic containing waste with domestic waste that contains elementary aluminium.

If wood impregnated with agents containing only copper is incinerated separately, then the ash will have a high content of this metal, probably considerably more than $10 \%$, and approaching the range in concentrate from copper ore. Boliden Mineral AB has extensive experience in benefitting copper from complex ores as well as from recycled material, and the possibilities of including ash rich in copper is presently being discussed.

The third fraction would constitute wood with a low content of chromium, copper and arsenic, and this fraction could readily be "co-incinerated" with a variety of other waste fuels, thus offering substantial advantages in terms of versatility.

Countries in which it is necessary to heat the buildings during a large part of the year have higher incentives than others for making investments in incineration facilities that use the heat efficiently and in such a way that health and the environment are well protected. Flow of matter, including segregation of contaminants is an integral part of such systems. Sorting of impregnated wood is feasible and can support separation, stabilization and recycling.

\section{Acknowledgements}

The present work has been supported by the Swedish Waste Management (Avfall Sverige - Swedish Waste Management). The author also wishes to thank the staff at EFO AB, Halmstad Energi och Miljö AB, and Söderenergi AB, as well as at Holger Andreasson AB, for enthusiastically sharing their knowledge.

\section{References}

[1] Swedish waste management 2014. (Swedish title: Svensk Avfallshantering 2014). Avfall Sverige - Swedish Waste Management, 2014.

[2] Statistics provided at the Internet page of District heating in Sweden (Swedish name: Svensk Fjärrvärme).

[3] Sjöblom, R., Sustainability of combustion and incineration of renewable fuels: example of Sweden. WIT Transactions on Ecology and The Environment, Vol. 176, pp. 173-184, 2013.

[4] Baccini, P. and Brunner, P. H., Metabolism of the anthroposphere: analysis, evaluation, design. MIT Press, 2nd edition, 2012.

[5] Sjöblom, R., Classification of ash as hazardous or non-hazardous waste. Crete 2014, 4th International Conference on Industrial and Hazardous Waste Management. Published by International Waste Working Group, 2014.

[6] Sjöblom, R., Classification of waste as hazardous or non-hazardous: the cases of ash and slag. WIT Transactions on Ecology and the Environment; Vol. 163, pp. 285-296, 2012.

[7] Sjöblom, R., Methods for analysing and sorting impregnated wood with regard to its content of chromium, copper and arsenic. (Swedish title: Mätmetodik och sorteringsteknik med avseende på krom, koppar och 
arsenik $\{C C A\}$ i träbaserade bränslen). Avfall Sverige - Swedish Waste Management report E2014:05, 2014.

[8] Callender, E., Heavy metals in the environment - historical trends. In Lollar B S, Editor. Environmental geochemistry. Treatise on geochemistry, Vol. 9. Elsevier, 2009.

[9] Kumpiene, J., Lagerkvist, A. and Maurice, C., Stabilization of $\mathrm{As}, \mathrm{Cr}, \mathrm{Cu}$, $\mathrm{Pb}$ and $\mathrm{Zn}$ in soil amendments: a review. Waste Management, Vol. 28, issue 1, pp. 215-225, 2008.

[10] Kumpiene, J., Desogus, P., Schulenburg, S., Arenella, M., Renella, G., Brännvall, E., Lagerkvist, A., Andreas, L. and Sjöblom, R., Utilisation of chemically stabilized arsenic-contaminated soil in a landfill cover. Environmental Science and Pollution Research. 20, 12, pp. 8649-8662, 2013.

[11] Kumpiene, J., Trace element immobilization in soil using amendments. In Hooda, P., Editor, Trace Elements in Soils. Chichester: Wiley-Blackwell, pp. 353-380, 2010.

[12] Thayer, J., S., Environmental chemistry of the heavy elements; Hydrido and organo compounds. VCH Publishers, 1995.

[13] Pauling, L., College chemistry. W. H. Freeman and company, San Francisco, 1957.

[14] Hamberg, N. P., Chemical investigations of the air in a domestic room with wallpaper containing arsenic. (Swedish title: Kemisk undersökning af luften i boningsrum, beklädda med arsenikhaltiga tapeter.) Nord. Med. Arkiv. Band. VI, Nr 3, pp. 1-21, 1874.

[15] Magel, G., Unzeitig, H., Heuss-Assbichler, S. and Fehr, K. T., Abiotic H2generation in a landfill example of Waldering. (German title: Abiotische H2-Produktion in NV-Monodeponien am Beispiel Waldering.) BayForrest-Forschungsvorhaben F188, Bayerischer Forschungsverbund Abfallsforschung und Reststoffverwertung 12, pp. 393-411, 2000).

[16] Burguera, M., Burguera, J., L., Brunetto, M., R., de la Guardia, M. and Salvador, A., Flow-injection atomic spectrometric determination of inorganic arsenic(III) and arsenic(V) species by use of an aluminiumcolumn arsine generator and cold-trapping arsine collection. Analytica Chimica Acta, 261, pp. 105-113, 1991.

[17] Jambeck, J., Dubey, B., Townsend, T. and Solo-Gabriele, H., Disposal of preservative-treated wood in landfills. In Townsend, T., G. and SoloGabriele, H., Editors, Environmental impacts of treated wood. Taylor \& Francis, pp. 447-466, 2006.

[18] Helsen, L. and Van den Bulck, E., Thermal treatment technologies for treated wood. In Townsend, T., G. and Solo-Gabriele, H., Editors, Environmental impacts of treated wood. Taylor \& Francis, pp. 447-466, 2006.

[19] Solo-Gabriele, H., Omar, A., Townsend, T. and Hahn, D., Identification of wood treated with waterborne metal-based preservatives. In Townsend, T., G. and Solo-Gabriele, H., Editors, Environmental impacts of treated wood. Taylor \& Francis, pp. 447-466, 2006. 